\title{
A POPULATION-SPECIFIC HTR2B STOP CODON PREDISPOSES TO SEVERE IMPULSIVITY
}

\author{
Laura Bevilacqua ${ }^{1}$, Stéphane Doly ${ }^{2}$, Jaakko Kaprio ${ }^{3,4,5}$, Qiaoping Yuan ${ }^{1}$, Roope Tikkanen ${ }^{6}$, \\ Tiina Paunio ${ }^{7}$, Zhifeng Zhou ${ }^{1}$, Juho Wedenoja ${ }^{8,9}$, Luc Maroteaux ${ }^{2}$, Silvina Diaz ${ }^{2}$, Arnaud \\ Belmer $^{2}$, Colin A. Hodgkinson ${ }^{1}$, Liliana Dell'Osso ${ }^{10}$, Jaana Suvisaari ${ }^{7}$, Emil Coccaro ${ }^{11}$, \\ Richard J Rose ${ }^{12}$, Leena Peltonen ${ }^{\star}, 8,9$, Matti Virkkunen ${ }^{6,13}$, and David Goldman ${ }^{1}$
}

${ }^{1}$ Laboratory of Neurogenetics, National Institute on Alcohol Abuse and Alcoholism, NIH, Rockville, MD, USA ${ }^{2}$ INSERM UMR-S 839 and Université Pierre et Marie Curie, Institut du Fer à Moulin, Paris, France ${ }^{3}$ Depaprtment of Public Health, University of Helsinki, Helsinki, Finland ${ }^{4}$ Institute for Molecular Medicine, Helsinki, Finland ${ }^{5}$ Unit for Child and Adolescent Psychiatry, National Institute for Health and Welfare, Helsinki, Finland ${ }^{6}$ Institute of Clinical Medicine,

Users may view, print, copy, download and text and data- mine the content in such documents, for the purposes of academic research, subject always to the full Conditions of use: http://www.nature.com/authors/editorial_policies/license.html\#terms

Correspondence should be addressed to David Goldman (davidgoldman@mail.nih.gov). .

*eena Peltonen is deceased. This study is dedicated to her memory and that of Markku Linnoila.

Supplementary Information: a figure summarizing the six components of this study is available in Supplementary Information. Author Contributions:

Laura Bevilacqua: Drafted manuscript. Revised manuscript. Conceptualization of study. Molecular analyses. Clinical analyses. Statistical analyses. Stéphane Doly: Behavioral analyses in mice. Statistical analyses. Jaakko Kaprio: Revised manuscript. Clinical analyses. Statistical analyses. Organization and support. Qiaoping Yuan: Statistical analyses. Roope Tikkanen: Clinical analyses. Tiina Paunio: Revised manuscript. Molecular analyses. Zhifeng Zhou: Molecular analyses. Juho Wedenoja: Molecular analyses. Luc Maroteaux: Revised manuscript. Conceptualization of study. Behavioral analyses in mice. Statistical analyses. Organization and support. Silvina Diaz: Behavioral analyses in mice. Statistical analyses. Arnaud Belmer: Behavioral analyses in mice. Statistical analyses. Colin A. Hodgkinson: Revised manuscript. Molecular analyses. Organization and support. Liliana Dell'Osso: Organization and support. Jaana Suvisaari: Revised manuscript. Clinical analyses. Emil Coccaro: Revised manuscript. Clinical analyses. Organization and support. Richard J Rose: Clinical analyses. Organization and support. Leena Peltonen: Clinical analyses. Organization and support. Matti Virkkunen: Revised manuscript. Clinical analyses. Organization and support. David Goldman: Drafted manuscript. Revised manuscript. Conceptualization of study. Molecular analyses. Clinical analyses. Statistical analyses. Organization and support.

This work was supported by the Intramural Research Program of the National Institute on Alcohol Abuse and Alcoholism, NIH and the Academy of Finland Centre of Excellence in Complex Disease Genetics. The Finntwin12 and Finntwin16 studies were supported by the National Institute on Alcohol Abuse and Alcoholism (AA-12502 and AA-09203 to RJR), and by the Academy of Finland $\left(100499,205585\right.$, and 118555 to JK). The studies on $\mathrm{Htr} 2 \mathrm{~b}^{-/-}$mice were supported by the Centre National de la Recherche Scientifique, the Institut National de la Santé et de la Recherche Médicale, the Université Pierre et Marie Curie, and by grants from the Fondation de France, the Fondation pour la Recherche Médicale, the French Ministry of Research (Agence Nationale pour la Recherche), and the European Union. LM's team is an "Equipe Fondation pour la Recherche Médicale". S. Diaz is supported by a fellowship from IBRO and Region Ile de France DIM STEM.

Author information: NCBI accession numbers for the HTR2B stop codon is: rs79874540. For all newly discovered SNPs NCBI accession numbers are listed in Supplementary Table 4.

Human studies. Written informed consent was obtained from each participant. Protocols were approved by the Institutional Review Board (IRB) of the National Institutes of Health and the National Institute of Mental Health (NIMH), by the Office for Protection from Research Risks (OPRR), Indiana University IRB, by the University of Helsinki Department of Psychiatry IRB, by the University of Helsinki Central Hospital IRB, the University of Turku Central Hospital IRB, and by the Ministry of Social Affairs and Health and the Ethics Committee of the National Public Health Institute of Finland.

Animal studies. Mice were housed under controlled environmental conditions. Behavioral tests and animal care were conducted in accordance with standard ethical guidelines (National Institutes of Health's "Guide for the Care and Use of Laboratory animals", and the European Communities Council European Communities Directive 86/609 EEC). All experiments involving mice were approved by the Ile de France Regional Ethics Committee for Animal Experiments. 
Department of Psychiatry, University of Helsinki, Helsinki, Finland ${ }^{7}$ Department of Psychiatry, Helsinki University Central Hospital, Helsinki, Finland ${ }^{8}$ Department of Medical Genetics, University of Helsinki, Helsinki, Finland ${ }^{9}$ Institute for Molecular Medicine Finland FIMM, University of Helsinki and National Institute for Health and Welfare, Helsinki, Finland ${ }^{10}$ Department of Psychiatry, University of Pisa, Italy ${ }^{11}$ Department of Psychiatry, The Pritzker School of Medicine, University of Chicago, Chicago, IL, USA ${ }^{12}$ Department of Psychological and Brain Sciences, Indiana University, Bloomington, Indiana ${ }^{13}$ Kellokoski Psychiatric Hospital, Kellokoski, Finland

\section{SUMMARY}

Impulsivity, describing action without foresight, is an important feature of several psychiatric diseases, suicidality and violent behavior. The complex origins of impulsivity hinder identification of the genes influencing both it and diseases with which it is associated. We performed exoncentric sequencing of impulsive individuals in a founder population, targeting fourteen genes belonging to the serotonin and dopamine domain. A stop codon in $H T R 2 B$ that is common (MAF $>1 \%$ ) but exclusive to Finns was identified. Expression of the gene in the human brain was assessed, as well as the molecular functionality of the stop codon that was associated with psychiatric diseases marked by impulsivity in both population and family-based analyses. Knockout of Htr $2 b$ increased impulsive behaviors in mice, indicative of predictive validity. Our study shows the potential for identifying and tracing effects of rare alleles in complex behavioral phenotypes using founder populations, and suggests a role for $H T R 2 B$ in impulsivity.

Impulsivity is a broad term describing behavior characterized by action without foresight, decreased inhibitory control and lack of consideration of consequences ${ }^{1}$. Cognitive function, attention and responses to reward are factors that are thought to contribute to the trait of impulsivity. Although impulsivity can be an adaptive dimension of personality, intolerance for delay, disinhibition, and the inappropriate weighting of contingencies are maladaptive ${ }^{2}$. The behavioral manifestations of impulsivity include suicide, addictions, Attention Deficit Hyperactivity Disorder (ADHD), and violent criminality ${ }^{3}$ as well as Antisocial Personality Disorder (ASPD), Borderline Personality Disorder (BPD) and Intermittent Explosive Disorder (IED). These behaviors and diagnoses, including impulsivity itself, are moderately heritable ${ }^{4,5}$, indicating that it should be feasible to identify genes influencing them. Gene identification would also validate the idea that it is possible to deconstruct the multi-process origins of impulsivity. Still, studies demonstrating that genetic variation predicts impulsivity have been relatively sparse ${ }^{6-11}$. The fact that few genes influencing impulsivity have been discovered could reflect the complexity of the phenotype, the nature of the samples or the methodologies used.

To detect novel alleles that influence impulsivity, we studied severely impulsive Finnish criminal offenders and matched controls. This study had six components (as charted in Supplementary Fig. 1): resequencing and identification of putatively functional variants in severe impulsive probands from a founder population, association and linkage with impulsive behavior, population genetics, evaluation of cognitive effects of the identified variant, gene expression and functionality, and animal studies. 
Exon-centric sequencing was performed on fourteen genes involved in dopamine or serotonin function (the genes are listed in Supplementary methods). Dysregulated activity of the monoamine neurotransmitters has been implicated in impulsivity both on a neuropharmacologic basis and on a genetic basis via gene knockouts and/or association studies with common functional variants. In rats, serotonin and dopamine interact in the control of impulsive choice, with differential actions in regions of the prefrontal cortex involved $^{12}$. The spontaneous impulsivity of rats correlates with lower levels of D2 receptors in the nucleus accumbens, predicting liability to compulsive drug seeking and addiction ${ }^{13}$, also in humans a reduction in D2 receptors, as well as a decrease in dopamine release, has been described in the ventral tegmental area of cocaine abusers ${ }^{14}$. The serotonin system has long been implicated in impulsivity ${ }^{15,16}$ and, in particular, impulsive aggression and suicide. MAOA knockout mice have higher levels of monoamines and increased aggressive behavior ${ }^{17}$, and a functional variable number tandem repeat in the $M A O A$ regulatory region (MAOA-LPR) moderates the effect of maltreatment on vulnerability to develop antisocial behavior ${ }^{8,18}$. Brunner et al had shown that a stop codon variant that produces complete deficiency of $M A O A$ activity co-segregated with severe impulsivity ${ }^{6}$. Stress-modified associations have been reported also for a polymorphism in the serotonin transporter (HTTLPR) to suicidality ${ }^{19,20}$.

Deep sequencing recently was successfully applied to gene identification in rare Mendelian disorders ${ }^{21}$. In the domain of complex disorders, sequencing revealed putatively functional alleles at a gene previously implicated by GWAS of Type 1 diabetes ${ }^{22}$. Here, we attempted to used sequencing to identify novel loci contributing to a non-Mendelian phenotype.

\section{Sequencing Finnish impulsive subjects}

Founder populations can increase power to detect effects of rare alleles. At autosomal loci, Finns are equally diverse as other Europeans, yet a restricted number of founders and isolation have molded the Finnish gene pool $^{23}$. Many disease alleles are more abundant, or unique, to Finland and conversely some disease alleles common in other European populations are rare or nonexistent ${ }^{23}$. From the standpoint of identifying rare or uncommon alleles with roles in complex phenotypes, it is perhaps most important that Finnish ancestry appears to have reduced the genetic heterogeneity of various diseases. For seventeen Finnish disease alleles, $70 \%$ of disease chromosomes (and as many as $98 \%$ for some diseases) were attributable to a single allele ${ }^{23}$.

Sequencing was conducted in 96 unrelated Finnish males with impulsive behavior and an equal number of unrelated Finnish males free of psychiatric diagnoses (Supplementary Table 1 and Methods). Probands had ASPD, BPD or IED and were all violent offenders and arsonists who, because of the extreme nature of their crimes, underwent inpatient forensic psychiatric examination at the University of Helsinki at the time of their initial incarceration. ASPD and BPD share genetic risk for impulsive aggression ${ }^{4}$, which is a central characteristic of both of these personality disorders. Impulsivity is also key to IED, described in the DSM-IV as a failure to resist aggressive impulses. 
The ninety-six cases were selected for resequencing from a larger Finnish violent offenders cohort comprising 228 cases on the basis that they had highest Brown-Goodwin Lifetime Aggression scores, 23.7 ( $\mathrm{SD} \pm 4.9)$ as compared to 8.1 ( $\mathrm{SD} \pm 4.9)$ in controls. Their higher scores were indicators of a life history of aggressive, violent, and impulsive behavior as behavioral manifestations of impulsive temperament. The ninety-six male controls were free of Axis I and II diagnoses and matched for age, and were selected for sequencing for SNP discovery from a larger control cohort comprising 295 individuals. As compared to controls, cases also had significantly higher impulsivity (action on the spur of the moment) scores on the Karolinska Scales of Personality $(\mathrm{p}<0.0001)^{24}$. However, analysis was conducted on a behaviorally based phenotype, rather than a measure of temperament, because behavior has repeatedly shown the strongest relationship to biological predictors including genes. Genetic loci previously implicated in impulsivity include the $M A O A$ stop codon linked to impulsive behavior in one Dutch family ${ }^{6}, H T T L P R$ at the serotonin transporter which predicts suicidality ${ }^{19,20}$ and the dopamine transporter VNTR which has been associated to ADHD ${ }^{11}$. Impulsive behavior also can be predicted by neurotransmitters and endocrine factors, as illustrated by associations of brain serotonin turnover ${ }^{25}$, testosterone levels and a gene/ testosterone interaction ${ }^{9}$. Animal behavioral pharmacology, gene knockout and straindifference studies all primarily rely on measured behavior. By selecting the most phenotypically extreme probands for sequencing, we increased the probability that we would detect functional variants altering impulsivity. Clinical and criminal records, including evaluation of premeditation and spontaneity of crimes, were available for all cases.

Exonic and promoter regions (comprising $82 \mathrm{~kb}$ ) were amplified in pools of 12 genomic DNAs and sequenced simultaneously at 80x coverage on an Illumina Genome Analyzer, as described in Methods. Sequencing allowed us to identify and accurately estimate frequencies of alleles (Supplementary Fig. 8 compares frequencies determined by sequencing and genotyping, $r=0.94$ ). Of 360 SNPs identified, 44\% were known (NCBI Build128). Frequencies of novel SNPs ranged as high as 0.2 . Within $37 \mathrm{~kb}$ of protein-coding DNA, 25 synonymous SNPs, of which 9 were novel, and 26 nonsynonymous SNPs (nsSNPs) were detected. Of a total of 22 nsSNPs confirmed by Sanger sequencing, 10 were novel.

\section{Association of putatively functional SNPs}

Four nsSNPs were predicted to be functional according to both SIFT (Sorting Intolerant From Tolerant) and PolyPhen (Polymorphism Phenotyping): TPH2 Pro206Ser (rs17110563), DRD1 Ser259Tyr, HTR2B Arg388Trp and HTR2B Q20*, a stop codon (Supplementary Table 5). These four nsSNPs were genotyped in male Finnish cases and controls. In a global test of association to an aggregate of potential susceptibility variants, these four putatively functional variants were twice as common in cases (13.0\%) compared to controls $\left(6.5 \%, \chi^{2}=6.76, p=0.009\right.$; Supplementary Table 6). However, this global association was driven by $H T R 2 B$ Q20*. 17/228 cases were heterozygous for $H T R 2 B * 20$ compared to $7 / 295$ controls $\left(\chi^{2}=7.26, p=0.007\right.$ ) (Supplementary Table 6 ), with an allele frequency in controls of 0.012 . Eighty-nine pedigrees comprising family members of the violent offenders were collected and all were genotyped without pre-selection for phenotype 
or genotype, identifying eight $H T R 2 B * 20$ carrier families (Fig. 1; Methods). Affected status was defined as presence of ASPD, BPD, or IED. The Transmission Disequilibrium Test detected over-transmission of $* 20$ to affected offspring $\left(\mathrm{McNemar} \chi^{2}=5.0, \mathrm{p}=0.025\right)$. Similarly, among affected individuals, $6 / 7$ had $* 20$ transmitted, and among unaffected individuals 10/14 did not have $* 20$ transmitted (Supplementary Table 7). From the cumulative binomial distribution, previously proposed for linkage of functional loci in families $^{26}$, the likelihood of $16 / 21$ or more linked outcomes was 0.013 .

The HTR2B gene is on 2q36.3-q37.1, a location implicated in early onset obsessive compulsive disorder ${ }^{27}$ and illicit substance abuse ${ }^{28}$. However, resequencing of $H T R 2 B$ in these two studies yielded no functional variants ${ }^{27,28}$. 5-HT2B receptor function in the brain is mainly unknown, however it has been shown that MDMA (Ecstasy) selectively binds and activates 5-HT2B receptors, inducing serotonin release, in mouse raphe nuclei leading to dopamine release in the nucleus accumbens and ventral tegmentum ${ }^{29}$, and HT2B agonists increase serotonin transporter phosphorylation ${ }^{30}$.

\section{HTR2B Q20* in humans}

We assessed molecular functionality of $H T R 2 B \mathrm{Q} 20^{*}$ by using RNA and proteins extracted from lymphoblastoid cell lines, and in addition $H T R 2 B$ expression was measured in multiple brain regions, including frontal cortex, by qPCR (Methods). *20 led to variable nonsensemediated RNA decay and blocked expression of the 5-HT2B receptor protein (Fig. 2, Methods). $H T R 2 B$ is widely expressed in the adult human brain, and the frontal lobe is one of the regions where it is most highly expressed (Methods, and Supplementary Fig. 13).

$H T R 2 B * 20$ is apparently exclusive to Finns. In $>3100$ individuals representative of worldwide diversity, including the Human Genome Diversity Panel (Supplementary Table 8 ) one additional $* 20$ carrier was observed: a female with a Finnish surname, and with alcoholism. Indicative of a common origin and founder population effect, $* 20$ was found on a single haplotype background (Supplementary Fig. 9), and in Finns who were likely to be non-admixed (Supplementary Fig. 2). Genetic sub-isolates have been identified within Finland, including families in Eastern Finland. Also, the Finnish population apparently was founded by two waves of migration: Eastern Uralic founders arrived 4000 years ago, followed by Indo-European speakers 2000 years later ${ }^{23}$. However, it is unlikely that the $* 20$ association is an occult admixture artifact because $* 20$ carriers are common across Finland (in Middle, Western and Eastern regions) (Supplementary Fig. 3), and cases and controls did not differ in Finnish ancestry (Supplementary Fig. 4, Methods).

In the 17 violent offenders (from the case/control study) who carried $* 20$, impulsivity played a strong role in their crimes. Although convicted for a variety of offenses including homicide, attempted homicide, arson, battery and assault, $94 \%$ of their crimes were committed under the influence of alcohol. The crimes of the $* 20$ carrier probands occurred as disproportionate reactions to minor irritations and were unpremeditated, without potential for financial gain, and recurrent. From court records up to an average age of 43, *20 carriers had committed an average of 5 violent crimes (range 2-12). The $* 20$ cases tended to fulfill criteria for ASPD (82\%) and IED (57\% meeting 3 of 4 IED criteria), except that alcoholism, 
ASPD and BPD are exclusionary for IED. Overall, $* 20$ carriers were cognitively normal (mean IQ: 98, SD: 14.9, range 75-124, two with IQ <87, Wechsler Adult Intelligence Scale).

In temperament, measured with the Tridimensional Personality Questionnaire, *20 carriers are, like others with ASPD, higher in Novelty Seeking and Harm Avoidance, but are otherwise more socially attached, empathic and dependent than the other violent offenders within the study group (Supplementary data). Extrapolating from the *20 frequency of 0.012 (and with $174 * 20$ carriers directly genotyped), 53,000 Finnish males (and as many females) are heterozygous. However, while few $* 20$ carriers are criminals, violent criminals with $* 20$ appear to represent some of the most impulsive individuals within our violent offender cohort. Among 100-155 homicides annually in the Finnish population of 5.3 million, there are few instances of multiple homicide. In our sample, only three individuals were convicted of multiple homicide, and all carried the $* 20$ allele.

In our sample, the influence of $* 20$ was not due to interaction with $M A O A$ or serotonin transporter genotypes (data not shown). However, it was not possible to rule out other gene interactions, or a modifying role of stress. CSF monoamine metabolite levels, another potential confound, did not differ in $* 20$ carriers (Supplementary data). Therefore, it is unlikely that their impulsivity was due to low turnover of serotonin, dopamine or norepinephrine or that $* 20$ substantially affects monoamine metabolism, as does the MAOA stop codon ${ }^{6}$.

Risk conferred by $* 20$ appears to be modulated by sex and alcohol. Worldwide, suicide accounts for $1.5 \%$ of deaths, and Finland has a very high suicide rate, especially among men $^{32}$. In our study, $70 \%$ of the $* 20$ male cases displayed impulsive suicidal behavior (e.g. slashings, hanging attempts, drug overdoses) usually while intoxicated, for an average of 3.2 suicide attempts. At age 33.5 ( $\mathrm{SD} \pm 11), 66 \%$ had at least one life-threatening suicide attempt. It is unknown if suicide risk conferred by $* 20$ extends to the general population, whose members are at lower risk. Males are more likely to commit suicide ${ }^{32}$ and to have ASPD and aggression, with a 10-fold higher preponderance for the early onset-life course persistent variant of ASPD $^{33}$. Moreover, alcohol-related violence is known to be higher among males, and the serotonin system is thought to contribute to individual differences in alcohol-facilitated impulsive aggression ${ }^{34}$.

In the violent offender cohort, *20 carriers were cognitively normal and in almost every instance acted out on their impulsivity only when inebriated. Having found the association of $* 20$ to impulsivity in a phenotypically extreme sample, it was important to define *20 frequency and relationship to behavior in the wider population, even though the only possible follow-up was in Finland. In >6000 Finns ascertained epidemiologically (rather from the criminal population), the $* 20$ allele frequency was 0.012 (the same as the frequency in controls) (Supplementary Table 9 ). We identified one $* 20$ homozygote, a young male adult with no major medical illness, but with a history of violent behavior whilst under the influence of alcohol (Supplementary methods).

We followed up cognitive effects of $* 20$ in 933 individuals in the FT12/FT16 Twin Studies (22 with the stop codon) (Supplementary methods). Overall, *20 carriers were again 
cognitively normal. However, male (but not female) $* 20$ carriers had significantly lower Digit Span Forward $(\mathrm{p}=0.002)$, and Backward $(\mathrm{p}<0.001)$ scores possibly indicating selective impairment in working memory (Supplementary Fig. 12), a specific measure of frontal cortical function.

\section{$\mathrm{Htr2b}^{-/-}$mice}

Although severe developmental consequences have been observed in Htr2b knockout mice, approximately $50 \%$ of the mice that survive the first postnatal week are apparently normal as adults ${ }^{35}$. These mice were reported to be impulsive in an open field novelty test ${ }^{29}$. We assessed Htr $2 b$ knockout mice for five separate measures of impulsivity and novelty seeking: delay discounting, activity in a novel environment, exposure to a novel object, motor activity after a dopamine D1 receptor agonist, and decreased latency to eat in the novelty suppressed feeding test (hyponeophagia). The $\mathrm{Htr} 2 \mathrm{~b}^{-/-}$mice were more impulsive and more responsive to novelty in all of these tests (Fig. 3). In rats, both impulsivity and response to novelty are predictors for the development of addiction-like behaviors ${ }^{36}$. In addition to their differences in behavior, $H \operatorname{tr} 2 b^{-/-}$males had a three-fold elevation in plasma testosterone (Fig. 3) (Supplementary methods). Testosterone (measured in the CSF of nine heterozygous violent offenders) also appeared to be higher in human males carrying *20 (Supplementary Fig. 11). This raises the possibility of interaction between $* 20$ and testosterone to contribute to impulsive behaviors, as was reported between MAOA and testosterone in the same population of Finns that we studied here?

\section{Discussion}

The aim of this study was to identify genetic variation associated with impulsivity, an intermediate phenotype thought to contribute to several psychiatric disorders, including addictions. ${ }^{12}$ The goal is to track shared genetic factors in these diseases and contribute to their reconceptualization on a neurobiological basis. Another purpose of identifying genes influencing impulsivity is to determine which of the potential etiologies and types of impulsivity, for example novelty seeking versus executive dysfunction ${ }^{36}$ are important in human populations. The discovery of genes influencing impulsive behavior would validate the idea that it is possible to deconstruct the multi-process origins of impulsive behavior.

$H T R 2 B * 20$ is associated and co-segregates with disorders characterized by impulsivity, reflected in severe crimes committed on the spur of the moment, as documented by criminal and clinical records, and under alcohol intoxication, a condition where impulse control is impaired. Thus the *20 allele can be regarded as one determinant of behavioral variation. However, presence of $* 20$ was not itself sufficient: male sex, testosterone level, the decision to drink alcohol, and probably other factors such as stress exposure, have important roles. Although relatively common in Finland, $H T R 2 B * 20$ is unlikely to explain a large fraction of the overall variance in impulsive behaviors. There are likely to be many pathways to impulsivity in its various manifestations, and the genetic association may be present only in the most phenotypically extreme. 
It is un-surprising that a stop codon variant discovered by sequencing within a founder population is common only in it, and even restricted to it. However, this observation is also in line with the significance of $* 20$ as a complete loss of function variant, and with the behavioral consequences in some heterozygous carriers. The relatively high frequency of *20 in Finns would thus reflect its status as a founder mutation, in contrast to MAOA, COMT and $H T T$ alleles that are common worldwide, that more modestly affect molecular function, and that may have counterbalancing selective advantages. However, it is highly unlikely that Finns are unique in possessing a severe genetic variation leading to impulsivity. There is the previous example of the MAOA stop codon, found in one Dutch family. On average, ten or more heterozygous stop codons reside in the genomes of each individual of European ancestry ${ }^{21}$ but perhaps because the source populations from which the probands sequenced did not have founder characteristics, no common stop codon had yet been reported for a neurotransmitter gene. Although rare variants identified in founder populations are more likely to be confined to those populations, analyses of the relationship between gene variation and phenotype can be conducted within the founder population, identifying new candidate genes and pathways influencing behavior or other etiologically complex phenotypes.

As has often been illustrated, the availability of mouse genetic models, including gene knockouts, offers an opportunity to test the predictive validity of genetic discoveries and to define effects in contexts where genetic background and environment are better controlled. The Htr $2 b$ mouse knockout reveals more general effects of $H T R 2 B$ deficiency on behavior, including effects on novelty seeking. This could be explained by pleiotropic actions of the serotonin 2B receptor. On the other hand, the effect of the Htr2b knockout on delay discounting appears to validate the effect of the $* 20$ stop codon on impulsivity in people. In people, we observed a significant association between the $H T R 2 B * 20$ variant and impairment in working memory, a neurocognitive process contributing or predictive of executive cognitive function. The ability to store and integrate knowledge about possible choices with current context enables the individual to select appropriate cognitive strategies and generate optimal reactions. This is coherent with the impulsivity observed in $H T R 2 B$ *20 cases who seemed deficient in ability to weigh the consequences of their acts.

The use of deep sequencing to detect a stop codon associated with impulsivity in a founder population reveals a role for the $H T R 2 B$ gene in behavior. It also suggests that this approach may be applicable to other complex behavioral traits, including those diseases for which impulsivity is itself an intermediate phenotype.

\section{METHODS SUMMARY}

Fourteen serotonergic and dopaminergic genes were resequenced (Solexa GA2) in 96 Finnish Caucasian male violent offenders and 96 matched controls free of psychiatric diagnosis. Exon-centric sequencing was performed by amplifying 108 regions, for a total of $82 \mathrm{~kb}$, in pools of 12 subjects. HTR2B Q20* was genotyped in a Finnish sample of 228 cases and 295 controls, in 89 Finnish families, and in 5684 individuals belonging to either a Finnish family dataset $(\mathrm{N}=1885)$, the Older Finnish Twin cohort $(\mathrm{N}=2388)$ and FinnTwin16 and FinnTwin12 studies $(\mathrm{N}=1411)$ as described in detail in supplementary 
methods, and in $>3100$ samples representing worldwide diversity. Genotyping was performed with a custom $5^{\prime}$ exonuclease assay (Applied Biosystems 7900) using these primers and probes:

- forward primer: 5'-AGAGTGTCTGAACTTCAAAGCACAA-3'

- reverse primer: 5'-TCCAGACCAGTTAGAAGAGATAACGT-3'

- $\quad$ robe 1: 5'-AGGTGCTCTGCAAAAT-3'

- $\quad$ robe 2: 5'-AGGTGCTCTACAAAAT-3'

186 ancestry informative markers were genotyped on 1536-SNP arrays (Illumina).

Quantitative PCR for HTR2B expression in thirteen human brain regions was determined by ABI Taqman gene expression assays (Hs01118766 and Hs00168362). B-actin was the internal control. Total protein and total RNA were extracted from lymphoblastoid cell lines using the TRIzol LS reagent protocol (Invitrogen). Nonsense-mediated RNA decay was detected by sequencing on a 3700ABI capillary sequencer cDNA from HTR2B Q20/*20 heterozygotes. HT2B protein was measured in 12 Finnish Q20/Q20 homozygotes and 14 Finnish Q20/*20 heterozygotes. Blots were probed with antisera raised against the Nterminal (mouse monoclonal Ab, Novus Biologicals), internal (goat polyclonal Ab, Santa Cruz Biotechnology) or C-terminal (rabbit polyclonal Ab, Santa Cruz Biotechnology) regions of the HT2B receptor, and GAPDH Ab (Millipore). Densitometry performed using NIH ImageJ. Htr $2 b^{-/-}$knockout mice were made in a pure 129Sv/PAS background and compared to 129/SvPAS control mice (8-10 week old) for four measures of response to novelty and for delay discounting.

\section{Supplementary Material}

Refer to Web version on PubMed Central for supplementary material.

\section{Acknowledgments}

We thank Longina Akhtar for assistance with tissue culture, Cheryl Marietta for assistance with measurement of receptor protein levels, Vibhuti Srivastava and Goli Yamini for helpful discussions, and Pei-Hong Shen for contributions to ancestry analyses. Monica Eggert and LaVonne Brown assisted with clinical ascertainment and assessment of the University of Helsinki sample. We thank Markku Linnoila for his contributions to the collection of the University of Helsinki sample. Elli Kempas assisted with genotyping. Jean-Marie Launay measured plasma testosterone levels in Htr2 $b^{-/-}$mice. We also thank Annamari Tuulio-Henriksson, Eero Vuoksimaa, Anja Häppölä, and Liisa Arala.

\section{Appendix \\ METHODS}

Finnish violent offenders cohort and controls

Cases were 228 unrelated Finnish male violent offenders and arsonists (Supplementary

Table 1) who, because of the extreme nature of their crimes, underwent forensic psychiatric examination at the time of their initial incarceration. They were studied as inpatients at the University of Helsinki ${ }^{37,25}$. These subjects were diagnosed with the Structural Clinical Interview for DSM (SCID) according to DSM-III-R criteria for ASPD, BPD and IED. 
Excluded were subjects with schizophrenia or a history of psychosis. Ninety-six cases were selected for resequencing from the larger Finnish case cohort, comprising 228 individuals with diagnosis of ASPD, BPD and IED, on the basis that they had highest Brown-Goodwin Lifetime Aggression (BGLAS) scores ${ }^{38}$ with scores of 23.7 ( $\mathrm{SD} \pm 4.9$ ) out of a theoretical maximum of 36. Controls $(\mathrm{N}=295)$ were unrelated, non-impulsive Finnish volunteers recruited by advertisements in local newspapers, paid for their participation and psychiatrically interviewed by trained psychiatrists. Cases and controls were independently blind-rated from interview data by two research psychiatrists under the supervision of a senior research psychiatrist. Inter-rater reliability was high, and differences were resolved by the senior psychiatrist. Controls were free of ASPD, BPD, IED, psychosis or schizophrenia but some had mood or anxiety disorders or alcohol use disorder (Supplementary Table 1). Ninety-six male controls free of Axis I and II diagnoses and matched for age were selected for sequencing for SNP discovery from a cohort of 295 controls. Controls had a BGLAS score of $8.1(\mathrm{SD} \pm 4.9)$.

A total of 89 pedigrees were collected. Family members were interviewed using the SCID and diagnosed using DSM-III-R criteria. DNA and data were available for 397 subjects in families.

Genomic DNA was prepared from lymphoblastoid cell lines.

\section{Resequencing}

Exon-centric targeting of $\mathbf{1 4}$ candidate genes-We custom-designed or used Applied Biosystem oligonucleotide primers to amplify 108 target regions that covered exons, flanking regions and $~ 800-1000$ bp of the upstream regions of 14 genes, for a total of $82 \mathrm{~kb}$ (Supplementary Table 2).

DNA pools-DNA samples were individually quantified in three replicates by RT-PCR, using TaqMan RNase P Detection Reagent kits (FAM) and Roche human DNA standards, and were normalized to $10 \mathrm{ng} / \mu \mathrm{L}$. Eight pools (12 subjects per pool) were made with equal amounts of DNA from 96 Finnish cases and in parallel fashion eight pools were made from 96 Finnish controls. Average sequencing coverage per individual per nucleotide was 80x.

DNA Amplification-DNA pools were amplified in 108 separate PCR reactions (Supplementary methods).

DNA Sequencing-Prior to sequencing, amplicon concentrations were normalized using SequalPrep Normalization Plate kits (Invitrogen). All amplicons from the same DNA pool were combined. The DNA was sheared by sonication and purified with QIAquick PCR purification kits (QIAGEN). Genomic DNA Preparation kits and protocol (Illumina) were used to prepare sequencing libraries.

Analysis of sequence data-Sequences were called from image files with the Illumina Genome Analyzer Pipeline and aligned to human reference sequence from NCBI build 36.3 using the Illumina Eland software. Each 36 base read was uniquely mapped to the human reference genome. Sequence reads with more than 2 mismatches were excluded. Sequence 
reads with alternative alleles that did not exactly match the reference genome did uniquely map to the corresponding location in the reference sequence. Additional results are described in supplementary data.

\section{Capillary electrophoresis sequencing}

nsSNPs were validated by Sanger sequencing using the BigDye Terminator Sequencing Mix (Applied Biosystems) and analyzed on the Applied Biosystems 3730 DNA Analyzer. Of 26 nsSNPs, 22 were validated, and overall 30/34 SNPs tested in this way were validated.

\section{Predicted Functionality}

Missense, nonsense and synonymous variants were predicted to be probably damaging or damaging for protein function via PolyPhen and SIFT amino acid substitution prediction methods. Four variants (DRD1 S259Y, HTR2B R388W, HTR2B Q20*, and TPH2 P206S rs 17110563) scored as damaging or intolerant by both methods were used in a global test of proportion of rare functional variants in cases (ASPD, BPD or IED) and controls. Genotypes of the four SNPs were collapsed so that an individual was coded as 1 if a rare allele was present and otherwise as 0 . Frequencies of putatively functional variants were globally compared between cases and controls, with the null hypothesis being a lack of difference between cases and controls in the proportion carrying the putatively functional variants. Case-control association test was also performed for HTR2B Q20* alone. Pearson $\chi^{2}$ test was used to test the null hypothesis. All analyses were conducted using JMP software v7.0 (SAS Institute, Cary, NC). Criterion for statistical significance was set at 0.05 .

\section{Genotyping}

HTR2B Q20* was genotyped in 228 Finnish cases and 295 Finnish controls and in 89 pedigrees belonging to the Finnish cohort for a total of 352 subjects. Taking into account that some families had affected probands, we genotyped a total of 872 Finnish DNAs. In addition to the Finnish case/control and family dataset and over 3100 samples representing worldwide diversity, we also genotyped a total of 5684 individuals belonging to either a Finnish family dataset $(\mathrm{N}=1885)$, or to the Older Finnish Twin cohort $(\mathrm{N}=2388)$ and FinnTwin16 and FinnTwin12 studies $(\mathrm{N}=1411)$ described in supplementary methods.

Genotyping of Q20* was performed with a custom 5' exonuclease assay (Applied Biosystems 7900) using these primers and probes:

- forward primer: 5'-AGAGTGTCTGAACTTCAAAGCACAA-3'

- reverse primer: 5'-TCCAGACCAGTTAGAAGAGATAACGT-3'

- probe 1: 5'-AGGTGCTCTGCAAAAT-3'

- $\quad$ probe 2: 5'-AGGTGCTCTACAAAAT-3'

\section{Ancestry Informative Markers (AIMs)}

A panel of 186 AIMs were genotyped on 1536-SNP arrays (Illumina) ${ }^{39}$. No difference was detected between cases (ASPD, BPD and IED) and controls in proportions of ancestries. The pattern of measured ancestry for seven ancestry factors derived separately for each subject 
was compared between controls $(\mathrm{N}=279)$ and cases $(\mathrm{N}=220)$ with reference to the Human Genome Diversity Panel (HGDP) (1051 DNAs representing 51 populations worldwide).

Finnish ancestry was measured using 177 ancestry informative markers in 29 Q20* carriers, 580 other Finns, and 200 individuals representing 10 European populations in HGDP. Principal component analysis was performed with EIGENSTRAT.

For HTR2B RNA and protein expression studies, total protein and RNA were extracted from lymphoblastoid cell lines using the TRIzol LS reagent protocol (Invitrogen).

\section{HTR2B cDNA Sequencing for nonsense-mediated decay}

Nonsense-mediated RNA decay was detected by sequencing cDNA from HTR2B Q20/*20 heterozygotes on a 3700ABI capillary sequencer (Fig. 2; Supplementary methods). The sequences of the upstream and downstream oligonucleotides were as follows: $5^{\prime}$ gagtgtttggcatggttaca- $3^{\prime}$ and $3^{\prime}$-accaggcaggacatagaaca- $5^{\prime}$ (Supplementary methods). HTR $2 B$ Q20 and *20 transcripts were quantitated by comparing the relative intensities of the Q20 and $* 20$ sequencing peaks within each heterozygous individual (Supplementary methods)

\section{Western Blots}

HT2B protein was measured in 12 Finnish Q20/Q20 homozygotes and 14 Finnish Q20/*20 heterozygotes. Western blots were prepared using 50 ug of protein per lane on a $10 \%$ BisTris gel (Invitrogen). Separated proteins were transferred to nitrocellulose using the iBlot transfer system (NuPage - Invitrogen). Blots were probed with antisera raised against the Nterminal (mouse monoclonal Ab, Novus Biologicals), internal (goat polyclonal Ab, Santa Cruz Biotechnology) or C-terminal (rabbit polyclonal Ab, Santa Cruz Biotechnology) regions of the HT2B receptor, and GAPDH Ab (Millipore).

Antibody binding was visualized on X-ray film (Kodak XAR) using chemiluminescence (ECL Plus, GE Healthcare). Densitometry performed using NIH ImageJ. Ratios between the 5-HT2B receptor and the housekeeping protein GAPDH were calculated to normalize 5HT2B protein quantity.

\section{Quantitative PCR for HTR2B in human brain}

Quantitative PCR for HTR2B expression in thirteen human brain regions was determined by ABI Taqman gene expression assays (Hs01118766 and Hs00168362). B-actin was the internal control.

\section{Neuropsychological assessment}

Neuropsychological assessment was conducted on both the combined FT16 and FT12 cohorts (described in Supplementary methods) for measures of verbal intellectual ability, working memory and executive function. Working memory was assessed with the Digit Span Forward and Backward subtests of the Wechsler Memory Scale-Revised (WMS-R). We analyzed the combined FT16 and FT12 datasets. A linear regression model was constructed using performance on the working memory test as the dependent variable and sex and genotype as independent variables. Sex was a significant predictor, so the sample 
was stratified into male and female. Male heterozygotes performed significantly worse on the Digit Span backward and forward tests, and combined score (Supplementary Table 12 and Supplementary Fig. 12). All statistical analyses were conducted using Stata (version 11, Stata Corp, College Station, Texas, USA). Criterion for statistical significance was set at 0.05. Bonferroni correction for multiple testing was applied, as presented in Supplementary Table 12 .

\section{Htr2b knockout mice}

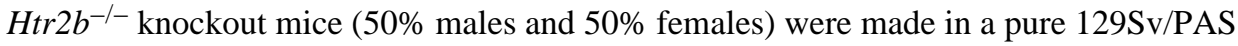
background. Wild type (WT) 129/SvPAS mice (8-10 week old), bred in-house, were used as controls.

Novelty seeking and impulsive behavior in $\mathrm{Htr} 2 \mathrm{~b}^{-/-}$knockout mice was investigated using five experimental measures: novelty-induced locomotion, locomotor reactivity in response to a dopamine D1 receptor agonist, exposure to a novel object, delay discounting and novelty-suppressed feeding. Plasma testosterone levels were measured.

\section{REFERENCES}

1. Winstanley CA, Eagle DM, Robbins TW. Behavioral models of impulsivity in relation to ADHD: translation between clinical and preclinical studies. Clin. Psychol. Rev. 2006; 26:379-395. [PubMed: 16504359]

2. Eysenck SB, Eysenck HJ. The place of impulsiveness in a dimensional system of personality description. Br. J. Soc. Clin. Psychol. 1977; 16:57-68. [PubMed: 843784]

3. DeJong J, Virkkunen M, Linnoila M. Factors associated with recidivism in a criminal population. J. Nerv. Ment. Dis. 1992; 180:543-550. [PubMed: 1522403]

4. Kendler KS, et al. The structure of genetic and environmental risk factors for DSM-IV personality disorders. Arch. Gen. Psychiatry. 2008; 65:1438-1446. [PubMed: 19047531]

5. Coccaro EF, Bergeman CS, McClearn GE. Heritability of irritable impulsiveness: a study of twins reared together and apart. Psychiatry Res. 1993; 48:229-249. [PubMed: 8272445]

6. Brunner HG, Nelen M, Breakefield XO, Ropers HH, van Oost BA. Abnormal behavior associated with a point mutation in the structural gene for monoamine oxidase A. Science. 1993; 262:578-580. [PubMed: 8211186]

7. Sabol SZ, Hu S, Hamer D. A functional polymorphism in the monoamine oxidase A gene promoter. Hum. Genet. 1998; 103:273-279. [PubMed: 9799080]

8. Caspi A, et al. Role of genotype in the cycle of violence in maltreated children. Science. 2002; 297:851-854. [PubMed: 12161658]

9. Sjöberg RL, et al. A non-additive interaction of a functional MAO-A VNTR and testosterone predicts antisocial behavior. Neuropsychopharmacology. 2008; 33:425-430. [PubMed: 17429405]

10. Misener VL, et al. Linkage of the dopamine receptor D1 gene to attention deficit/hyperactivity disorder. Mol Psychiatry. 2004; 9:500-509. [PubMed: 14569274]

11. Faraone SV, et al. Molecular genetics of attention deficit/hyperactivity disorder. Biol. Psychiatry. 2005; 57:1313-1323. [PubMed: 15950004]

12. Winstanley CA, et al. Double dissociation between serotonergic and dopaminergic modulation of medial prefrontal and orbitofrontal cortex during a test of impulsive choice. Cereb. Cortex. 2006; 16:106-114. [PubMed: 15829733]

13. Everitt BJ, et al. Review. Neural mechanisms underlying the vulnerability to develop compulsive drug-seeking habits and addiction. Philos. Trans. R. Soc. Lond. B. Biol. Sci. 2008; 363:31253135. [PubMed: 18640910] 
14. Volkow ND, Fowler JS, Wang GJ. Role of dopamine in drug reinforcement and addiction in humans: results from imaging studies. Behav. Pharmacol. 2002; 13:355-366. [PubMed: 12394411]

15. Virkkunen M, Linnoila M. Brain serotonin, type II alcoholism and impulsive violence. J. Stud. Alcohol. Suppl. 1993; 11:163-169. [PubMed: 8410958]

16. Chiavegatto $S$, et al. Brain serotonin dysfunction accounts for aggression in male mice lacking neuronal nitric oxide synthase. Proc. Natl. Acad. Sci. 2001; 98:1277-1281. [PubMed: 11158630]

17. Cases O, et al. Plasma membrane transporters of serotonin, dopamine, and norepinephrine mediate serotonin accumulation in atypical locations in the developing brain of monoamine oxidase A knock-outs. J. Neurosci. 1998; 18:6914-6927. [PubMed: 9712661]

18. Ducci F, et al. Interaction between a functional MAOA locus and childhood sexual abuse predicts alcoholism and antisocial personality disorder in adult women. Mol. Psychiatry. 2007; 13:334347. [PubMed: 17592478]

19. Roy A, Hu XZ, Janal MN, Goldman D. Interaction between childhood trauma and serotonin transporter gene variation in suicide. Neuropsychopharmacology. 2007; 32:2046-2052. [PubMed: 17356577]

20. Caspi A, et al. Influence of life stress on depression: moderation by a polymorphism in the 5-HTT gene. Science. 2003; 301:386-389. [PubMed: 12869766]

21. Ng SB, et al. Targeted capture and massively parallel sequencing of 12 human exomes. Nature. 2009; 461:272-276. [PubMed: 19684571]

22. Nejentsev S, Walker N, Riches D, Egholm M, Todd JA. Rare variants of IFIH1, a gene implicated in antiviral responses, protect against type 1 diabetes. Science. 2009; 324:387-389. [PubMed: 19264985]

23. Peltonen L, Jalanko A, Varilo T. Molecular genetics of the Finnish disease heritage. Hum. Mol. Genet. 1999; 8:1913-1923. [PubMed: 10469845]

24. Gustavsson JP. Swedish universities Scales of Personality (SSP): construction, internal consistency and normative data. Acta. Psychiatr. Scand. 2000; 102:217-225. [PubMed: 11008858]

25. Virkkunen M, et al. CSF biochemistries, glucose metabolism, and diurnal activity rhythms in alcoholic, violent offenders, fire setters, and healthy volunteers. Arch. Gen. Psychiatry. 1994; 51:20-27. [PubMed: 7506515]

26. Abel L, Alcais A, Mallet A. Comparison of four sib-pair linkage methods for analyzing sibships with more than two affecteds: Interest of the binomial maximum likelihood approach. Genetic Epidemiology. 1998; 15:371-390. [PubMed: 9671987]

27. Kim SJ, et al. Mutation screening of human 5-HT(2B) receptor gene in early-onset obsessivecompulsive disorder. Mol. Cell. Probes. 2000; 14:47-52. [PubMed: 10722792]

28. Lin Z, Walther D, Yu XY, Drgon T, Uhl GR. The human serotonin receptor 2B: coding region polymorphisms and association with vulnerability to illegal drug abuse. Pharmacogenetics. 2004; 14:805-811. [PubMed: 15608559]

29. Doly S, et al. Serotonin 5-HT2B receptors are required for 3,4 methylenedioxymethamphetamineinduced hyperlocomotion and 5-HT release in vivo and in vitro. J. Neurosci. 2008; 28:2933-2940. [PubMed: 18337424]

30. Launay JM, Schneider B, Loric S, Da Prada M, Kellermann O. Serotonin transport and serotonin transporter-mediated antidepressant recognition are controlled by 5-HT2B receptor signaling in serotonergic neuronal cells. FASEB J. 2006; 20:1843-1854. [PubMed: 16940156]

31. Blanpain C, et al. Serotonin 5-HT(2B) receptor loss of function mutation in a patient with fenfluramine-associated primary pulmonary hypertension. Cardiovasc. Res. 2003; 60:518-528. [PubMed: 14659797]

32. Hawton K, van Heeringen K. Suicide. Lancet. 2009; 373:1372-1381. [PubMed: 19376453]

33. Rutter M, Caspi A, Moffitt TE. Using sex differences in psychopathology to study causal mechanisms: unifying issues and research strategies. J. Child. Psychol. Psychiatry. 2003; 44:10921115. [PubMed: 14626453]

34. Chiavegatto S, Quadros IM, Ambar G, Miczek K,A. Individual vulnerability to escalated aggressive behavior by a low dose of alcohol: decreased serotonin receptor mRNA in the prefrontal cortex of male mice. Genes Brain Behav. 2010; 9:110-119. [PubMed: 20002201] 
35. Jaffré F, et al. Serotonin and angiotensin receptors in cardiac fibroblasts coregulate adrenergicdependent cardiac hypertrophy. Circ. Res. 2009; 104:113-23. [PubMed: 19023134]

36. Belin D, Mar AC, Dalley JW, Robbins TW, Everitt BJ. High impulsivity predicts the switch to compulsive cocaine-taking. Science. 2008; 6:1352-1355. [PubMed: 18535246]

37. Linnoila M, et al. Low cerebrospinal fluid 5-hydroxyindoleacetic acid concentration differentiates impulsive from non impulsive violent behavior. Life Sci. 1983; 33:2609-2614. [PubMed: 6198573]

38. Brown GL, et al. Aggression in humans correlates with cerebrospinal fluid amine metabolites. Psych. Res. 1979; 1:131-139.

39. Hodgkinson CA, et al. Addictions biology: haplotype-based analysis for 130 candidate genes on a single array. Alcohol Alcohol. 2008; 43:505-515. [PubMed: 18477577] 

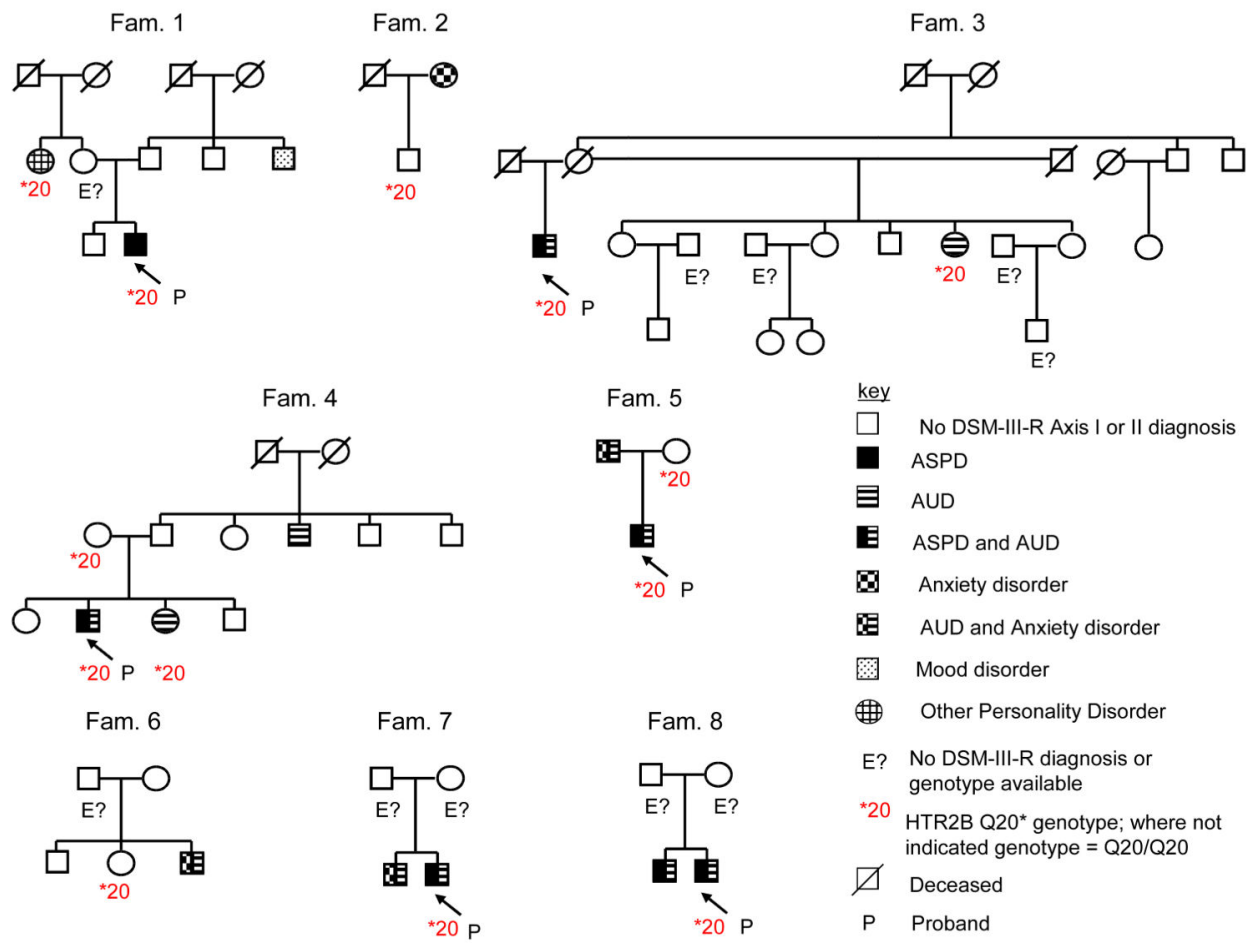

Fam. 5

key

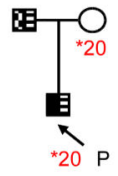

$\square \quad$ No DSM-III-R Axis I or II diagnosis

- ASPD

目 AUD

E ASPD and AUD

Q Anxiety disorder

A AUD and Anxiety disorder

Mood disorder

Other Personality Disorder

Fam. 8

E? No DSM-III-R diagnosis or genotype available

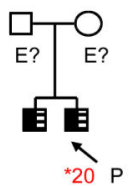

${ }^{*} 20$ HTR2B Q20* genotype; where not indicated genotype $=$ Q20/Q20

$\triangle$ Deceased

$\mathrm{P}$ Proband

Fig.1. HTR2B $* 20$ co-segregates with impulsivity

Co-segregation of $H T R 2 B * 20$ with ASPD and AUD in eight informative families. 
a

b
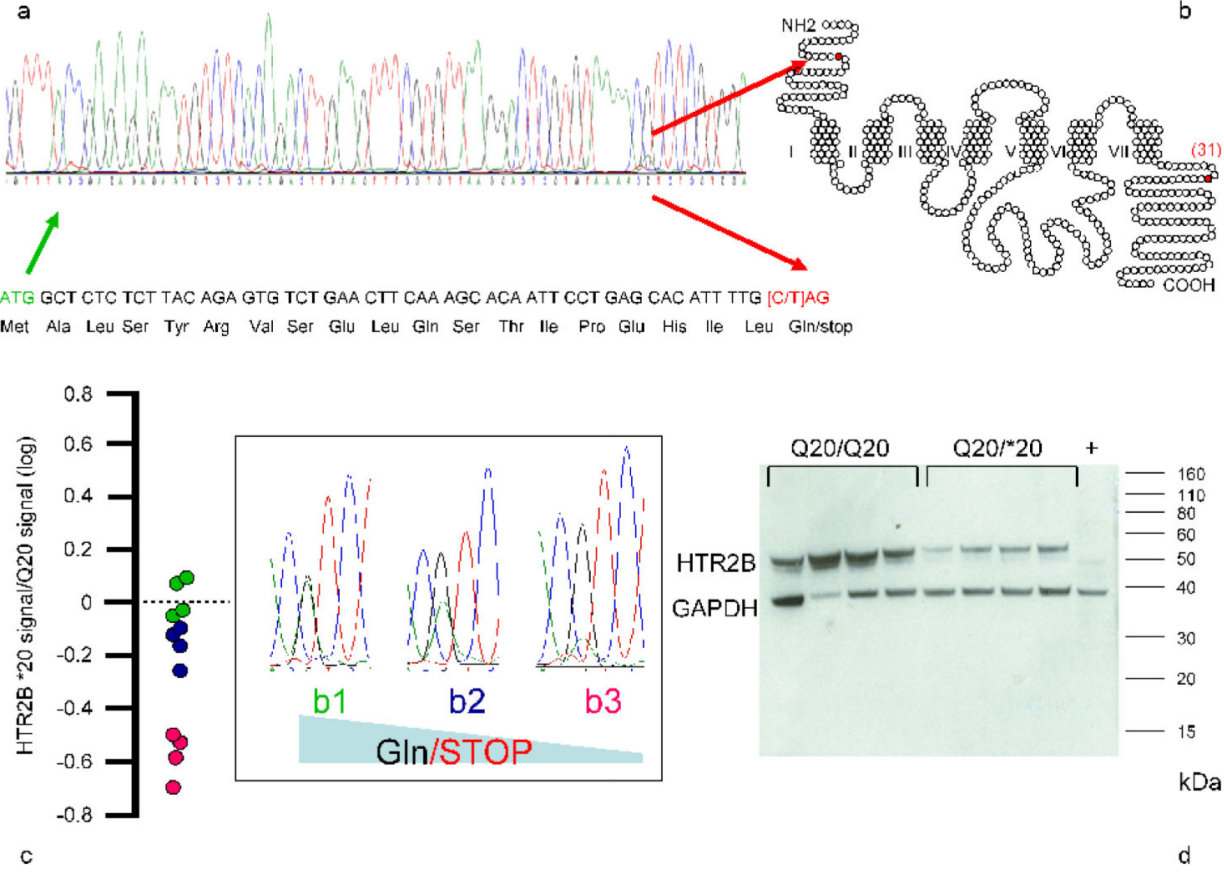

Fig. 2. HTR2B $* \mathbf{2 0}$ blocks protein expression

a) CDNA and b) protein locations of $H T R 2 B$ Q20*. c) Variable stop codon-mediated RNA decay determined by cDNA sequencing of twelve $* 20$ heterozygotes. d) $* 20$-mediated blockade of 5-HT2B protein expression in Western blots (validated with three anti-5-HT2B antibodies - described in Methods). The 5-HT2B protein ratio was 1.93: 1 in fourteen Q20/Q20 homozygotes (mean 1.78, SD 2.24) compared to 14 Q20/*20 heterozygotes (mean 0.92 , SD 1.14) $(\mathrm{p}=0.03)$ (Methods). 
a

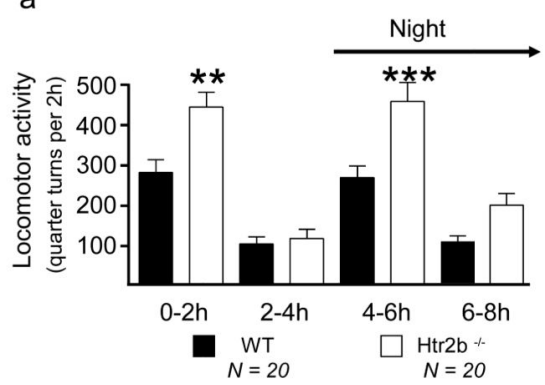

C

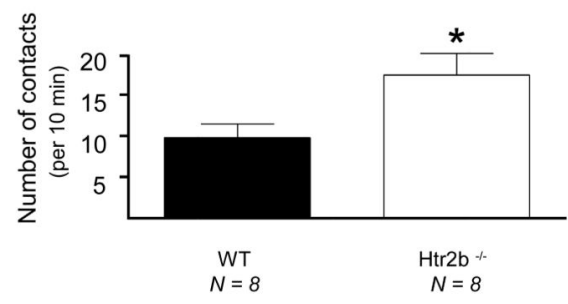

e

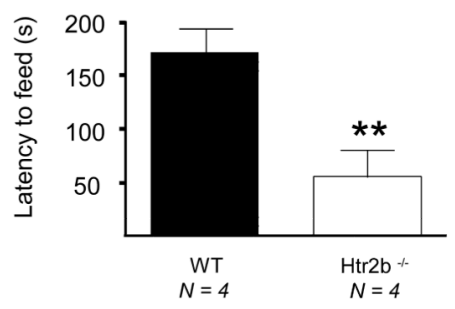

b

SKF $812974 \mathrm{mg} / \mathrm{kg}$

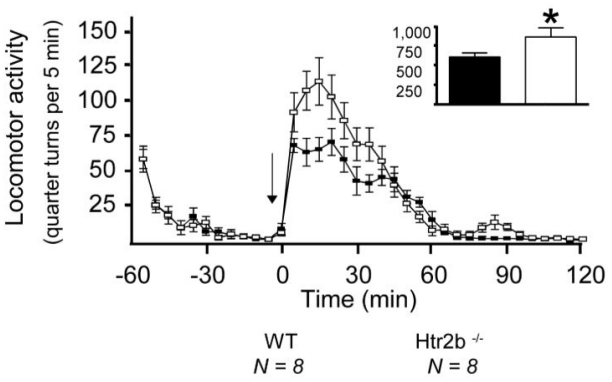

d

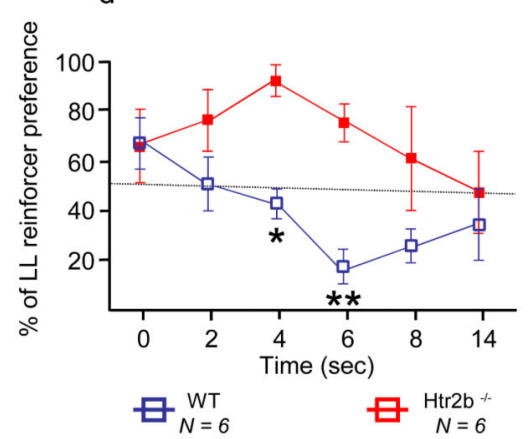

f

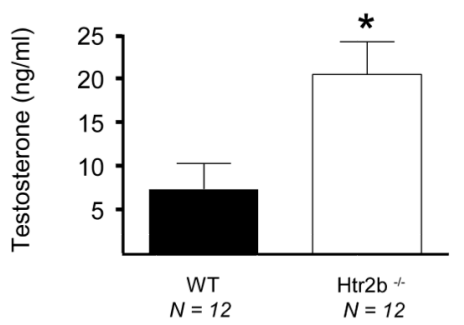

Fig.3. Increased impulsivity and novelty seeking in $\mathrm{Htr} 2 b^{-/-}$mice

a) Increased locomotor response of $\mathrm{Htr} 2 \mathrm{~b}^{-/-}$mice to environmental novelty and b) to dopamine D1 receptor agonists. c) Increased number of contacts of Htr $2 b^{-/-}$mice with a novel object. d) Increased delay-discounting of $H t r 2 b^{-/-}$mice. e) Reduced hyponeophagia in 18-hr starved Htr2 $\mathrm{b}^{-/-}$mice. f) Male $\mathrm{Htr} 2 \mathrm{~b}^{-/-}$mice have three-fold higher plasma testosterone levels as compared to control mice. * $\mathrm{p}<.05, * * \mathrm{p}<.01, * * * \mathrm{p}<.001$. 